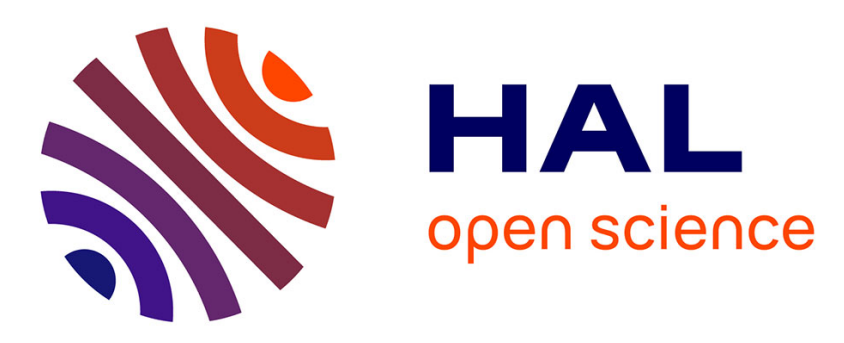

\title{
General Formula for Event-Based Stabilization of Nonlinear Systems with Delays in the State
}

Sylvain Durand, Nicolas Marchand, Jose Fermi Guerrero Castellanos

\section{To cite this version:}

Sylvain Durand, Nicolas Marchand, Jose Fermi Guerrero Castellanos. General Formula for EventBased Stabilization of Nonlinear Systems with Delays in the State. Delays and Networked Control Systems, 6, Springer, pp.59-77, 2016, Advances in Delays and Dynamics, 978-3-319-32372-5. 10.1007/978-3-319-32372-5_4. hal-01330818

\section{HAL Id: hal-01330818 https://hal.science/hal-01330818}

Submitted on 13 Jun 2016

HAL is a multi-disciplinary open access archive for the deposit and dissemination of scientific research documents, whether they are published or not. The documents may come from teaching and research institutions in France or abroad, or from public or private research centers.
L'archive ouverte pluridisciplinaire HAL, est destinée au dépôt et à la diffusion de documents scientifiques de niveau recherche, publiés ou non, émanant des établissements d'enseignement et de recherche français ou étrangers, des laboratoires publics ou privés. 


\title{
General Formula for Event-based Stabilization of Nonlinear Systems with Delays in the State
}

\author{
Sylvain Durand, Nicolas Marchand and J. Fermi Guerrero-Castellanos
}

\begin{abstract}
In this chapter, a universal formula is proposed for event-based stabilization of nonlinear systems affine in the control and with delays in the state. The feedback is derived from the seminal law proposed by E. Sontag (1989) and then extended to event-based control of affine nonlinear undelayed systems. Under the assumption of the existence of a control Lyapunov-Krasovskii functional (CLKF), the proposal enables smooth (except at the origin) asymptotic stabilization while ensuring that the sampling intervals do not contract to zero. Global asymptotic stability is obtained under the small control property assumption. Moreover, the control can be proved to be smooth anywhere under certain conditions. Simulation results highlight the ability of the proposed formula. The particular linear case is also discussed.
\end{abstract}

\section{Introduction}

The control synthesis problem is quite complex for systems with non linearities, particularly when the control laws have to be implemented on a real-time platform. Different techniques exist. The most classical way to address a discrete-time feedback for nonlinear systems is i) to implement a (periodic) continuous-time control algorithm with a sufficiently small sampling period. This procedure is denoted as emulation. However, the hardware used to sample and hold the plant measurements

Sylvain Durand

ICube, INSA Strasbourg, Univ. Strasbourg, CNRS (UMR 7357), Strasbourg, France

e-mail: sylvainedurandchamontin. fr

Nicolas Marchand

GIPSA-Lab, Univ. Grenoble Alpes, CNRS (UMR 5216), Grenoble, France

e-mail: nicolas.marchandegipsa-lab.fr

J. Fermi Guerrero-Castellanos

Autonomous University of Puebla (BUAP), Faculty of Electronics, Puebla, Mexico

e-mail: fguerrerodece.buap.mx 
or compute the feedback control action may make impossible the reduce of the sampling period to a level that guarantees acceptable closed-loop performance, as demonstrated in [15]. Furthermore, although periodicity simplifies the design and analysis, it results in a conservative usage of resources. Other methods are ii) the application of sampled-data control algorithms based on an approximated discretetime model of the process, like in [26], or iii) the modification of a continuous-time stabilizing control using a general formula to obtain a redesigned control suitable for sampled-data implementation, as done in [25]. But all these techniques are not generic enough for engineering applications. Finally, iv) event-triggered control approaches have also been suggested as a solution in recent decades, where the control law is event-driven. These novel alternatives are resource-aware implementations, they overcome drawbacks of emulation, redesigned control and complexity of the underlying nonlinear sampled-data models.

Whereas the control law is computed and updated at the same rate regardless whether is really required or not in the classical time-triggered approaches, the event-based paradigm relaxes the periodicity of computations and communications in calling for resources whenever they are indeed necessary (for instance when the dynamics of the controlled system varies). This is clearly an opportunity for embedded and networked control systems. Nevertheless, although eventbased control is well-motivated, only few works report theoretical results about stability, convergence and performance. Typical event-detection mechanisms are functions on the variation of the state (or at least the output) of the system, like in $[3,4,6,7,9,14,23,29,30]$. It has notably been shown in [4] that the control law can be updated less frequently than with a periodic scheme while still ensuring the same performance. Stabilization of linear and nonlinear systems is analyzed in $[1,8,24,34,35]$, where the events are related to the variation of a Lyapunov function or the time derivative of a Lyapunov function (and consequently to the state too). On the other hand, only few works deal with time-delay systems (which are of high concern in networked systems and in general for cyber-physical systems). One can refer to $[6,13,21,22]$ for linear systems for instance. As evidenced by the above reviewed literature, very little attention has been dedicated to the stabilization of nonlinear time-delayed systems using an event-based approach. To the authors' knowledge, this is the first time that an event-based control strategy is proposed.

Technically, it has been shown that if a control Lyapunov function (CLF) is known for a nonlinear system that is affine in the control, then the CLF and the system equations can be used to redesign the feedback by means of so-called universal formulas. These formulas are called universal because they depend only upon the CLF and the system equations, and not on the structure of those equations. The concept of CLF is therefore a useful tool for synthesizing robust control laws for nonlinear systems. In particular, the present work is based on the Sontag's universal formula [33], which event-based version was recently proposed in [24] for undelayed systems. The combination of i) an event function (based on the time derivative of the CLF) and ii) a feedback function (that is only updated when the event function vanishes) ensures the strict decrease of the CLF and consequently the asymptotic stability of the closed-loop system. For time-delay systems, the idea 
of CLF has been extended in the form of control Lyapunov-Razumikhin functions (CLRF) and control Lyapunov-Krasovskii functionals (CLKF), see [16-18]. The latter form is more flexible and easier to construct than CLRFs. Moreover, if a CLKF is known for a nonlinear time-delay system, several stabilizing control laws can be constructed using universal formulas derived for CLFs (such as the Sontag's one for instance) to achieve global asymptotic stability of the closed-loop system. Accordingly, the universal event-based formula developed in [24] for undelayed systems is extended here for the stabilization of affine nonlinear time-delay systems using CLKF. The present work extends the results previously presented in [8]. The class of time-delay systems under consideration is restricted to depend on some discrete delays and a distributed delay. Moreover, only state delays are considered (delays in the control signal, i.e. input delays, are not concerned).

The rest of the document is organized as follows. In section 2, preliminaries on classical (time-triggered) stabilization of nonlinear time-delay systems are presented. CLF and CLKF definitions are recalled as well as well-known universal formulas. The main contribution is then detailed in section 3. The event-based paradigm is firstly introduced and then a universal event-based formula, based on the Sontag's formula, is proposed for the stabilization of affine nonlinear systems with delays in the state. The smooth control particular case is also treated. Illustrative examples are given for both nonlinear and linear cases. A discussion finally concludes the chapter. Proofs are given in Appendix.

\section{Preliminaries on nonlinear time-delay system stabilization}

Stability is an important issue in control theory. For nonlinear dynamical systems, this is mainly treated with the theory of Lyapunov: if the derivative of a Lyapunov function candidate (a scalar positive definite function of the states) can be shown to be negative definite along the trajectories of a given system, then the system is guaranteed to be asymptotically stable [19]. For closed-loop systems, this means to propose a feedback function and then search for an appropriate Lyapunov function or, inversely, propose a Lyapunov function candidate and then find a feedback strategy that renders its derivative negative [19]. Nevertheless, it can be difficult to find a Lyapunov function candidate or even to determine whether or not one exists. Obviously, some techniques can help for such Lyapunov-based control synthesis.

\subsection{Control Lyapunov function}

The (Lyapunov-based) control synthesis problem was made more formal with the introduction of control Lyapunov function (CLF) $[2,32]$ for systems affine in the control input. A CLF is a (smooth) positive definite, radially unbounded function, which derivative can be made negative definite at each state (except possibly at the 
origin) by some feasible input. In addition, one may require that the CLF fulfils the small control property for global stability.

To summarize, let consider the affine nonlinear dynamical system

$$
\begin{aligned}
& \dot{x}(t)=f(x(t))+g(x(t)) u(t) \\
& \text { with } \quad x(0):=x_{0}
\end{aligned}
$$

with $x(t) \in \mathcal{X} \subset \mathbb{R}^{n}$ and $u(t) \in \mathcal{U} \subset \mathbb{R}^{m}$ the state and input (control) space vectors. $f: \mathcal{X} \rightarrow \mathcal{X}$ and $g: \mathcal{X} \rightarrow \mathbb{R}^{n \times m}$ are smooth functions with $f$ vanishing at the origin. Also, let define $\mathcal{X}^{*}:=\mathcal{X} \backslash\{0\}$ hereafter. Note that only null stabilization is considered here and the dependence on $t$ can be omitted in the sequel for the sake of simplicity.

Definition 1 (Control Lyapunov function [33]). A smooth and positive definite functional $V: \mathcal{X} \rightarrow \mathbb{R}$ is a control Lyapunov function (CLF) for system (1) if for each $x \neq 0$ there is some $u \in \mathcal{U}$ such that

$$
\begin{aligned}
& \alpha(x)+\beta(x) u<0 \\
& \text { with } \mid \begin{array}{l}
\alpha(x):=L_{f} V(x)=\frac{\partial V}{\partial x} f(x) \\
\beta(x):=L_{g} V(x)=\frac{\partial V}{\partial x} g(x)
\end{array}
\end{aligned}
$$

where $L_{f} V$ and $L_{g} V$ are the Lie derivatives of $f$ and $g$ functions respectively.

Property 1 (Small control property [33]). If for any $\mu>0, \varepsilon>0$ and $x$ in the ball $\mathcal{B}(\mu) \backslash\{0\}$, there is some $u$ with $\|u\| \leq \varepsilon$ such that inequality (2) holds, then it is possible to design a feedback control that asymptotically stabilizes the system.

Furthermore, it has been shown that if a CLF is known for a nonlinear system that is affine in the control, then the CLF and the system equations can be used to find some so-called universal formulas that render the system asymptotically stable. Several known universal formulas exist, in particular Sontag's [33] and Freeman's [10] formulas are presented in the sequel. Other methods, like the domination redesign formula [31] is also discussed but it will not be treated in details here.

\subsection{Control Lyapunov-Krasovskii functionals}

For (nonlinear) time-delay systems, there exist two main Lyapunov techniques, called the Krasovskii method of Lyapunov functionals [20] and the Razumikhin method of Lyapunov functions [28]. Motivated by the concept of CLF and the role it plays in robust stabilization of nonlinear systems, these methods have also been extended in the form of control Lyapunov-Razumikhin functions (CLRF) [16] and control Lyapunov-Krasovskii functionals (CLKF) [17]. 
Several stabilizing control laws can be constructed to achieve global asymptotic stability of the closed-loop system using one of the universal formulas derived for CLFs. For instance, Sontag's [33] and Freeman's [10] formulas apply for CLKF [17] whereas the domination redesign formula [31] applies for CLRF [16]. Note that this latter formula also applies for an augmented CLKF, as shown in [18]. Moreover, the CLKF form is more flexible and easier to construct than CLRFs. For these reasons, only Krasovskii methods are detailed in the sequel (but the proposal can be easily extended to the Razumikhin version).

Hereafter, the state of a time-delay system is described by $x_{d}:[-r, 0] \rightarrow \mathcal{X}$ defined by $x_{d}(t)(\theta)=x(t+\theta)$. This notation, used in [17] in particular, seems more convenient than the more conventional $x_{t}(\theta)$. Note that the dependence on $t$ and $\theta$ can be omitted in the sequel for the sake of simplicity, writing $x_{d}(\theta)-$ or only $x_{d}$ - instead of $x_{d}(t)(\theta)$ for instance. Let consider the affine (in the control) nonlinear dynamical time-delay system

$$
\begin{aligned}
& \dot{x}=f\left(x_{d}\right)+g\left(x_{d}\right) u \\
& \text { with } \quad x_{d}(0)(\theta):=\chi_{0}(\theta)
\end{aligned}
$$

where $f: \mathcal{X} \rightarrow \mathcal{X}, g: \mathcal{X} \rightarrow \mathbb{R}^{n \times m}$ are smooth functions and $\chi_{0}:[-r, 0] \rightarrow \mathcal{X}$ is a given initial condition.

Remark 1. Input delays of the form $u(t-\tau)$ are not considered in this chapter. However, the control law is computed using the state $x_{d}$ of the time-delay system.

Note that the class of time-delay systems under consideration in this paper is restricted to depend on $l$ discrete delays and a distributed delay in the form

$$
\begin{aligned}
& \dot{x}=\Phi\left(x_{\tau}\right)+g\left(x_{\tau}\right) u \\
& \text { with } \quad \Phi\left(x_{\tau}\right):=f_{0}\left(x_{\tau}\right)+\int_{-r}^{0} \Gamma(\theta) F\left(x_{\tau}, x(t+\theta)\right) d \theta \\
& \text { and } \quad x_{\tau}:=\left[x, x\left(t-\tau_{1}\right), x\left(t-\tau_{2}\right), \ldots, x\left(t-\tau_{l}\right)\right]
\end{aligned}
$$

where $f_{0}: \mathcal{X} \rightarrow \mathcal{X}, g: \mathcal{X} \rightarrow \mathbb{R}^{n \times m}$ and $F: \mathbb{R}^{(l+2) n} \rightarrow \mathbb{R}^{\Gamma}$ are smooth functions of their arguments. Without loss of generality, it is assumed that $F\left(x_{\tau}, 0\right)=0$ and the matrix $\Gamma:[-r, 0] \rightarrow \mathbb{R}^{n \times \Gamma}$ is piecewise continuous (hence, integrable) and bounded.

Definition 2 (Control Lyapunov-Krasovskii functional [17]). Let defined a smooth functional $V: \mathcal{X} \rightarrow \mathbb{R}$ of the particular form

$$
\begin{array}{l|l}
V\left(x_{d}\right)=V_{1}(x)+V_{2}\left(x_{d}\right)+V_{3}\left(x_{d}\right) \\
\text { with } & \begin{array}{l}
V_{2}\left(x_{d}\right)=\sum_{j=1}^{l} \int_{-\tau_{j}}^{0} S_{j}(x(t-\varsigma)) d \varsigma \\
V_{3}\left(x_{d}\right)=\int_{-r}^{0} \int_{t+\theta}^{t} L(\theta, x(\varsigma)) d \varsigma d \theta
\end{array}
\end{array}
$$


where $V_{1}$ is a smooth, positive definite, radially unbounded function of the current state $x$ (i.e. the classical control Lyapunov function for undelayed systems, as defined in Definition 1), $V_{2}$ and $V_{3}$ are non-negative functionals respectively due to the discrete delays and the distributed delay in (4), $S_{j}: \mathcal{X} \rightarrow \mathbb{R}$ and $L: \mathbb{R}^{+} \times \mathcal{X} \rightarrow \mathbb{R}$ are non-negative integrable functions, smooth in the $x$-argument. Then $V$ in (5) is a control Lyapunov-Krasovskii functional (CLKF) for system (4) if there exists a function $\lambda$, with $\lambda(s)>0$ for $s>0$, and two class $\mathcal{K}_{\infty}$ functions $\kappa_{1}$ and $\kappa_{2}$ such that

$$
\kappa_{1}\left(\left|\chi_{0}\right|\right) \leq V\left(\chi_{d}\right) \leq \kappa_{2}\left(\left\|\chi_{d}\right\|\right)
$$

and (see Remark 3 for the definition of $L_{f}^{*} V$ )

$$
\begin{aligned}
& \beta_{d}\left(\chi_{d}\right)=0 \Rightarrow \alpha_{d}\left(\chi_{d}\right) \leq-\lambda\left(\left|\chi_{0}\right|\right) \\
& \text { with } \mid \begin{array}{l}
\alpha_{d}\left(x_{d}\right):=L_{f}^{*} V\left(x_{d}\right) \\
\beta_{d}\left(x_{d}\right):=L_{g} V_{1}\left(x_{d}\right)
\end{array}
\end{aligned}
$$

for all piecewise continuous functions $\chi_{d}:[-r, 0] \rightarrow \mathcal{X}$, where $\chi_{0}$ is defined in (3).

Remark 2. The restriction on the class of delay systems (4) and the corresponding particular CLKF (5) is needed to avoid the problems that arise due to noncompactness of closed bounded sets in the space $(C([-r, 0], \mathcal{X}),\|\cdot\|)$, where $C([-r, 0], \mathcal{X})$ denotes the space of continuous functions from $[-r, 0]$ into $\mathcal{X}$. This is discussed in [16,17].

Remark 3. Whereas the classical Lie derivative notation is used in $L_{g} V_{1}(x)=$ $\frac{\partial V_{1}}{\partial x} g(x)$ for the CLKF part $V_{1}$ which is function of the current state $x$, an extended Lie derivative is required for functionals of the form (5). $L_{f}^{*} V$, initially defined in [17], comes from the time derivative of the CLKF V in (5) along trajectories of the system (4), that is

$$
\begin{aligned}
\dot{V}=L_{f}^{*} V\left(x_{d}\right)+ & L_{g} V_{1}\left(x_{d}\right) u=\alpha_{d}\left(x_{d}\right)+\beta_{d}\left(x_{d}\right) u \\
\text { with } \quad L_{f}^{*} V\left(x_{d}\right):= & \frac{\partial V_{1}}{\partial x} \Phi+\sum_{j=1}^{l}\left(S_{j}(x)-S_{j}\left(x\left(t-\tau_{j}\right)\right)\right) \\
& +\int_{-r}^{0}(L(\theta, x)-L(\theta, x(t+\theta))) d \theta
\end{aligned}
$$

where $\Phi$ is defined in (4). 


\subsection{Universal formulas for the stabilization of affine nonlinear time-delay systems}

Universal formulas derived for CLFs have been extended for the stabilization of affine nonlinear time-delay systems (4) with a CLKF of the form (5). In particular, the Sontag's [33] and Freeman's [10] versions are detailed here.

Theorem 1 (Sontag's universal formula with CLKF [17]). Assume that system (4) admits a CLKF of the form (5). For any real analytic function $q: \mathbb{R} \rightarrow \mathbb{R}$ such that $q(0)=0$ and $b q(b)>0$ for $b \neq 0$, let $\phi_{s}: \mathbb{R}^{2} \rightarrow \mathbb{R}$ be defined by

$$
\phi_{s}(a, b):= \begin{cases}\frac{a+\sqrt{a^{2}+b q(b)}}{b} & \text { if } b \neq 0 \\ 0 & \text { if } b=0\end{cases}
$$

Then, the feedback $u: \mathcal{X} \rightarrow \mathcal{U}$, smooth on $\mathcal{X}^{*}$, defined by

$$
u\left(x_{d}\right):=-\beta_{d}\left(x_{\tau}\right) \phi_{s}\left(\alpha_{d}\left(x_{d}\right),\left\|\beta_{d}\left(x_{d}\right)\right\|^{2}\right)
$$

with $x_{\tau}$ and $\alpha_{d}, \beta_{d}$ defined in (4) and (6) respectively, is such that (6) is satisfied for all non zero piecewise continuous functions $\chi_{d}:[-r, 0] \rightarrow \mathcal{X}$.

Theorem 2 (Freeman's universal formula with CLKF [17]). Assume that system (4) admits a CLKF of the form (5). For any continuous and positive definite function $\eta: \mathbb{R}^{2} \rightarrow \mathbb{R}$, let $\phi_{f}: \mathbb{R}^{2} \rightarrow \mathbb{R}$ be defined by

$$
\phi_{f}(a, b):=\left\{\begin{array}{lll}
\frac{a+\eta(a, b)}{b} & \text { if } \quad a+\eta(a, b)>0 \\
0 & \text { if } a+\eta(a, b) \leq 0
\end{array}\right.
$$

Then, the feedback $u: \mathcal{X} \rightarrow \mathcal{U}$, smooth on $\mathcal{X}^{*}$, defined by

$$
u\left(x_{d}\right):=-\beta_{d}\left(x_{\tau}\right) \phi_{f}\left(\alpha_{d}\left(x_{d}\right),\left\|\beta_{d}\left(x_{d}\right)\right\|^{2}\right)
$$

with $x_{\tau}$ and $\alpha_{d}, \beta_{d}$ defined in (4) and (6) respectively, is such that (6) is satisfied for all non zero piecewise continuous functions $\chi_{d}:[-r, 0] \rightarrow \mathcal{X}$.

Property 2 (Small control property with CLKF [17]). If the CLKF $V$ in Theorem 1 of Theorem 2 satisfies the small control property, then the control is continuous at the origin and so is globally asymptotically stable the closed-loop system.

Remark 4. Choosing the function $\eta(10)$ as the particular form

$$
\eta(a, b)=\sqrt{a^{2}+b q(b)}
$$

where $q$ is a continuous, positive semidefinite function, gives the same function (8) as originally proposed by Sontag. 
Remark 5. As already said, the domination redisign formula [31] has also been extended for time-delay systems using CLRF in [16] and CLKF in [18]. The feedback $u: \mathcal{X} \rightarrow \mathcal{U}$, smooth on $\mathcal{X}^{*}$, takes the more general form

$$
u\left(x_{d}\right):=-\beta_{d}\left(x_{\tau}\right) \phi_{d}\left(V\left(x_{d}\right)\right)
$$

where the scalar function $\phi_{d}$ is called the dominating function. Also, a particular choice of this function can lead to the original Sontag's function (8).

\section{Event-based stabilization of nonlinear time-delay systems}

The idea behind extending the (time-triggered) universal formulas to event-driven versions is to obtain equivalent but resource-aware strategies, because the control signal will be computed and updated only when a certain condition is satisfied in the event-based case. This was already done in [24] for the undelayed case and it is extended here for time-delay systems. The event-based paradigm is firstly introduced. Then, an event-based formula for the stabilization of affine nonlinear time-delay systems admitting a CLKF is then detailed, derived from the Sontag's formula [33]. Other universal formulas are not concerned but the extension is trivial since they are all similarly constructed. An illustrative example highlights the ability of the proposal. Finally, the particular case of linear systems is discussed.

\subsection{Event-based formalization}

The classical discrete-time framework of controlled systems consists in sampling the system uniformly in time with a constant sampling period. Although periodicity simplifies the design and analysis, it results in a conservative usage of resources (computation, communication, energy) since the control law is computed and updated at the same rate regardless it is really required or not. Fortunately, some innovative works addressed resource-aware implementations of the control law, where the control law is event driven (when a certain condition is satisfied).

Definition 3 (Event-based feedback). By event-based feedback we mean a set of two functions, that are

i) an event function $\epsilon: \mathcal{X} \times \mathcal{X} \rightarrow \mathbb{R}$ that indicates if one needs (when $\epsilon \leq 0$ ) or not (when $\epsilon>0$ ) to recompute the control law;

ii) a feedback function $v: \mathcal{X} \rightarrow \mathcal{U}$.

The solution of (1) with event-based feedback $(\epsilon, v)$ starting in $x_{0}$ at $t=0$ is then defined as the solution of the differential system

$$
\dot{x}(t)=f(x(t))+g(x(t)) v\left(t_{i}\right) \quad \forall t \in\left[t_{i}, t_{i+1}[\right.
$$


where the time instants $t_{i}$, with $i \in \mathbb{N}$, are considered as events (they are determined when the event function $\epsilon$ vanishes and denote the sampling time instants). Also let define $x_{i}$ the memory of the state value at the last event, that is

$$
x_{i}:=x\left(t_{i}\right)
$$

With such a formalization, the control value is updated each time $\epsilon$ becomes negative. Usually, one tries to design an event-based feedback so that $\epsilon$ cannot remain negative (and so is updated the control only punctually). In addition, one also wants that two events are separated with a non vanishing time interval avoiding the Zeno phenomenon. All these properties are encompassed with the Minimal interSampling Interval (MSI) property introduced in [24]. In particular:

Property 3 (Semi-uniformly MSI). An event-triggered feedback is said to be semiuniformly MSI if and only if the inter-execution times can be below bounded by some non zero minimal sampling interval $\underline{\tau}(\delta)>0$ for any $\delta>0$ and any initial condition $x_{0}$ in the ball $\mathcal{B}(\delta)$ centered at the origin and of radius $\delta$.

Remark 6. A semi-uniformly MSI event-driven control is a piecewise constant control with non zero sampling intervals (useful for implementation purpose).

A particular event-based feedback has already been proposed in [24] for the stabilization of affine nonlinear undelayed systems, based on the Sontag's universal formula [33]. The idea is to have a control law $v$ quite similar to the one in the classical approach and an event function $\epsilon$ related to the time derivative of the CLF in order to ensure a (global) asymptotic stability of the closed-loop system. In the present chapter, such an event-based feedback is extended for the stabilization of affine nonlinear systems with time delay using CLKF. In the sequel, let

$$
x_{d i}:=x_{d}\left(t_{i}\right)
$$

be the memory of the delayed state value at the last event, by analogy with (14).

\subsection{Event-based stabilization of nonlinear time-delay systems}

Based on the Sontag's universal formula with CLKF previously introduced in Theorem 1, an event-based feedback (see Definition 3) that asymptotically stabilizes affine nonlinear time-delay systems is proposed here.

Theorem 3 (Event-based universal formula with CLKF). If there exists a CLKF $V$ of the form (5) for system (4), then the event-based feedback $(\epsilon, v)$ defined by

$$
\begin{aligned}
& v\left(x_{d}\right)=-\beta_{d}\left(x_{\tau}\right) \Delta\left(x_{\tau}\right) \gamma\left(x_{d}\right) \\
& \epsilon\left(x_{d}, x_{d i}\right)=-\alpha_{d}\left(x_{d}\right)-\beta_{d}\left(x_{d}\right) v\left(x_{d i}\right) \\
& \quad-\sigma \sqrt{\alpha_{d}\left(x_{d}\right)^{2}+\Omega\left(x_{d}\right) \beta_{d}\left(x_{d}\right) \Delta\left(x_{d}\right) \beta_{d}\left(x_{d}\right)^{T}}
\end{aligned}
$$


with

- $\alpha_{d}$ and $\beta_{d}$ as defined in (6);

- $\Delta: \mathcal{X}^{*} \rightarrow \mathbb{R}^{m \times m}$ (a tunable parameter) and $\Omega: \mathcal{X} \rightarrow \mathbb{R}$ are smooth positive definite functions ;

- $\gamma: \mathcal{X} \rightarrow \mathbb{R}$ defined by

$$
\gamma\left(x_{d}\right):= \begin{cases}\frac{\alpha_{d}\left(x_{d}\right)+\sqrt{\alpha_{d}\left(x_{d}\right)^{2}+\Omega\left(x_{d}\right) \beta_{d}\left(x_{d}\right) \Delta\left(x_{d}\right) \beta_{d}\left(x_{d}\right)^{T}}}{\beta_{d}\left(x_{d}\right) \Delta\left(x_{d}\right) \beta_{d}\left(x_{d}\right)^{T}} & \text { if } x_{d} \in \mathcal{S}_{d} \\ 0 & \text { if } x_{d} \notin \mathcal{S}_{d}\end{cases}
$$

with $\mathcal{S}_{d}:=\left\{x_{d} \in \mathcal{X} \mid\left\|\beta_{d}\left(x_{d}\right)\right\| \neq 0\right\} ;$

- $\sigma \in[0,1[$ a tunable parameter ;

where $x_{d i}$ and $x_{\tau}$ are defined in (15) and (4) respectively, is semi-uniformly MSI, smooth on $\mathcal{X}^{*}$ and such that the time derivative of $V$ satisfies (6) $\forall x \in \mathcal{X}^{*}$.

Remark 7. The simplification made with respect to the original result in [24] (for the stabilization of nonlinear undelayed systems) resides in the assumptions made for the functions $\Omega$ and $\Delta$, that are more restrictive here whereas they are assumed to be definite only on the set $\mathcal{S}_{d}$ in the original work.

Remark 8. The idea behind the construction of the event-based feedback (16)-(17) is to compare the time derivative of the CLKF $V$ i) in the event-based case, that is when applying the piecewise feedback $v\left(x_{d i}\right)$, and ii) in the classical case, that is when applying $v\left(x_{d}\right)$ instead of $v\left(x_{d i}\right)$. The event function is the weighted difference between both, where $\sigma$ is the weighted value. By construction, an event is enforced when the event function $\epsilon$ vanishes to zero, that is hence when the stability of the event-based scheme does not behave as the one in the classical case. Also, the convergence will be faster with higher $\sigma$ but with more frequent events in return. $\sigma=0$ means updating the control when $\dot{V}=0$.

Property 4 (Global asymptotic stability). If the CLKF $V$ in Theorem 3 satisfies the small control property, then the event-based feedback (16)-(17) is continuous at the origin and so is globally asymptotically stable the closed-loop system.

Property 5 (Smooth control). If there exists some smooth function $\omega: \mathcal{X} \rightarrow \mathbb{R}^{+}$ such that on $\mathcal{S}_{d}^{*}:=\mathcal{S}_{d} \backslash\{0\}$

$$
\omega\left(x_{d}\right) \beta_{d}\left(x_{d}\right) \Delta\left(x_{d}\right) \beta_{d}\left(x_{d}\right)^{T}-\alpha_{d}\left(x_{d}\right)>0
$$

then the control is smooth on $\mathcal{X}$ as soon as $\Omega\left(x_{d}\right)\left\|\Delta\left(x_{d}\right)\right\|$ vanishes at the origin with

$$
\Omega\left(x_{d}\right):=\omega\left(x_{d}\right)^{2} \beta_{d}\left(x_{d}\right) \Delta\left(x_{d}\right) \beta_{d}\left(x_{d}\right)^{T}-2 \alpha_{d}\left(x_{d}\right) \omega\left(x_{d}\right)
$$

Proof. All proofs are given in the Appendix section. 


\section{Example}

Consider the nonlinear time-delay system

$$
\begin{aligned}
& \dot{x}_{1}=u \\
& \dot{x}_{2}=-x_{2}+x_{2 d}+x_{1}^{3}+u \\
& \text { with } \quad x_{2 d}:=x_{2}(t-\tau)
\end{aligned}
$$

that admits a CLKF (proposed in [17])

$$
\begin{aligned}
& V(x)=\frac{1}{2}\left(x_{1}^{2}+x_{2}^{2}\right)+\frac{1}{2} \int_{-\tau}^{0} x_{2 d}^{2}(\theta) d \theta \\
& \text { with } \quad \begin{array}{l}
\alpha_{d}=x_{2}\left(-x_{2}+x_{2 d}+x_{1}^{3}\right)+\frac{1}{2}\left(x_{2}^{2}-x_{2 d}^{2}\right) \\
\beta_{d}=x_{1}+x_{2}
\end{array}
\end{aligned}
$$

Indeed, setting $\lambda(|x|)=\frac{1}{4}|x|^{4}$ yields

$$
\begin{aligned}
& \beta_{d}=0 \Rightarrow x_{1}=-x_{2} \\
& \Rightarrow \alpha_{d}=-\frac{1}{2}\left(x_{2}-x_{2 d}\right)^{2}-x_{2}^{4} \leq-x_{2}^{4} \leq-\lambda(|x|)
\end{aligned}
$$

which proves that (22) is a CLKF for (21) using Definition 2.

The time evolution of $x, v(x)$ and the event function $\epsilon\left(x, x_{i}\right)$ is depicted in Fig. 1, for $\Delta=I_{n}$ (the identity matrix), $\Omega(x)$ is as defined in (20) (for smooth control everywhere), with $\omega=0.1, \sigma=0.6, x_{0}=[0.5-1]^{T}$ and a time delay $\tau=2 s$. One could remark that only 7 events occurs in the $50 \mathrm{~s}$ simulation time (including the first event at $t=0$ ) when applying the proposed event-based approach (16)-(17). Furthermore, $x_{1}$ and $x_{2}$ rapidly converge to 0 with the first 4 events.

\subsection{Particular case of linear systems}

Consider the simple linear system with single delay $\tau$

$$
\dot{x}(t)=A x(t)+A_{d} x(t-\tau)+B u(t)
$$

Take $P$ and $S$ the positive definite matrices solution of the linear matrix inequality (LMI) given by

$$
\left[\begin{array}{cc}
A^{T} P+P A-4 \rho P B R^{-1} B^{T} P+S & P A_{d}-4 \rho P B R^{-1} B^{T} P \\
A_{d}^{T} P-4 \rho P B R^{-1} B^{T} P & -S
\end{array}\right]<0
$$

where $R$ is positive definite matrix, and $\rho>0$, are tunable parameters. Then the Lyapunov-Krasovskii functional $V$ defined by 

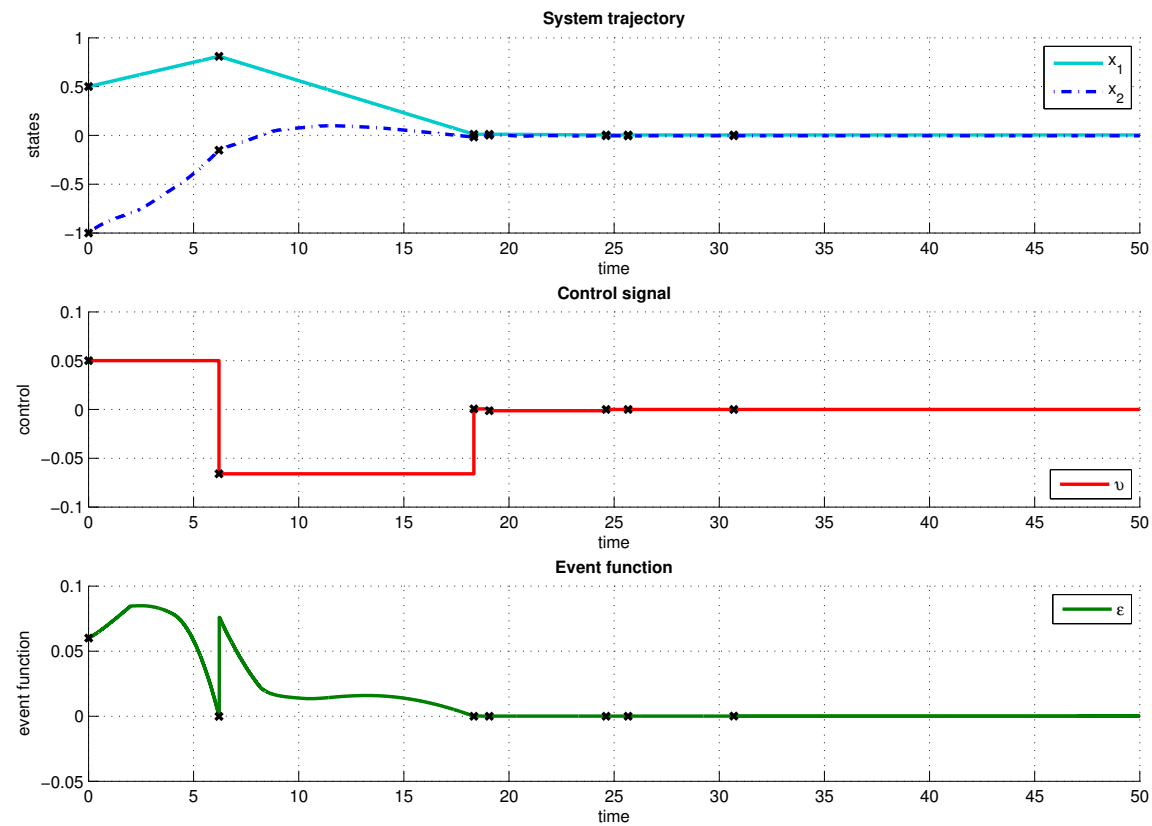

Fig. 1 Simulation results of system (21) with CLKF as in (22) and event-based feedback (16)-(17).

$$
V\left(x_{d}\right)=x^{T}(t) P x(t)+\int_{\tau}^{0} x_{d}(\theta)^{T} S x_{d}(\theta) d \theta
$$

is a CLKF for system (23) since for all $x, u=-2 \rho B^{T} P x$ renders the time derivative of $V$ strictly negative for $x \neq 0$.

Remark 9. The particular delay-independent form (25) has been proposed for system (23) without control input. More complex delay-dependent forms also exist in the literature but are not concerned here, see [11,12,27] for instance for further details.

Remark 10. Remember the first right-hand term in (25) is the classical CLF for a linear system without delay, whereas the second term is added for a single delay. The third term in the general CLKF form (5) is not needed in the present case without distributed delay.

The (extended) Lie derivatives are then obtained from the expressions in (6)-(7), that yields 


$$
\begin{aligned}
\alpha_{d}\left(x_{d}\right) & =\left[\begin{array}{c}
x \\
x_{d}
\end{array}\right]^{T}\left[\begin{array}{cc}
A^{T} P+P A+S & P A_{d} \\
A_{d}^{T} P & -S
\end{array}\right]\left[\begin{array}{c}
x \\
x_{d}
\end{array}\right] \\
\beta_{d}\left(x_{d}\right) & =2\left[\begin{array}{c}
x \\
x_{d}
\end{array}\right]^{T^{T}} P B
\end{aligned}
$$

Then, with $\Omega\left(x_{d}\right)$ according to (20) for the tunable parameters defined by $\Delta=R^{-1}$ and $\omega=\rho$, the control given by

$$
v\left(x_{d}\right)=-\omega \Delta\left[\begin{array}{c}
\beta(x)^{T} \\
\beta\left(x_{d}\right)^{T}
\end{array}\right]
$$

is smooth everywhere and linear. The event function given by

$$
\epsilon\left(x_{d}, x_{d i}\right)=(\sigma-1) \alpha_{d}\left(x_{d}\right)+\omega \beta_{d}\left(x_{d}\right) \Delta\left[\begin{array}{c}
\beta\left(x_{i}-\sigma x\right)^{T} \\
\beta\left(x_{d i}-\sigma x_{d}\right)^{T}
\end{array}\right]
$$

is linear.

\section{Conclusion}

In this chapter, an extension of the Sontag's universal formula was proposed for event-based stabilization of affine nonlinear systems with delays in the state. Whereas the original work deals with control Lyapunov functions for the case of undelayed systems, some control Lyapunov-Krosovsky functionals (CLKF) are now required for a global (except at the origin) asymptotic stabilization of time-delay systems. The sampling intervals do not contract to zero, avoiding Zeno phenomena. Moreover, the control is continuous at the origin if the CLKF fulfills the small control property. With additional assumption, the control can be proved to be smooth everywhere. Simulation results were provided, highlighting the low frequency of control updates. The linear case was also discussed.

Next step is to test the proposal in a real-time implementation and also consider input delays. Another way of investigation could be to develop general universal event-based formulas for nonlinear (time-delay) systems, in the spirit of [5].

\section{Appendix}

Proofs of the present contribution were previously presented in [8]. They are recalled here. 


\section{Proof of Theorem 3}

The proof follows the one developed in [24] for event-based control of systems without delays (1). First, let define hereafter

$$
\psi(x):=\sqrt{\alpha_{d}(x)^{2}+\Omega(x) \beta_{d}(x) \Delta(x) \beta_{d}(x)^{T}}
$$

Let begin establishing $\gamma$ is smooth on $\mathcal{X}^{*}$. For this, consider the algebraic equation

$$
P\left(x_{d}, \zeta\right):=\beta_{d}\left(x_{d}\right) \Delta\left(x_{d}\right) \beta_{d}\left(x_{d}\right)^{T} \zeta^{2}-2 \alpha_{d}\left(x_{d}\right) \zeta-\Omega\left(x_{d}\right)=0
$$

Note first that $\zeta=\gamma(x)$ is a solution of (30) for all $x_{d} \in \mathcal{X}$. It is easy to prove that the partial derivative of $P$ with respect to $\zeta$ is always strictly positive on $\mathcal{X}^{*}$

$$
\frac{\partial P}{\partial \zeta}:=2 \beta_{d}\left(x_{d}\right) \Delta\left(x_{d}\right) \beta_{d}\left(x_{d}\right)^{T} \zeta-2 \alpha_{d}\left(x_{d}\right)
$$

Indeed, when $\left\|\beta_{d}\left(x_{d}\right)\right\|=0$, (6) gives $\frac{\partial P}{\partial \zeta}=-2 \alpha_{d}\left(x_{d}\right) \geq 2 \lambda\left(\left|\chi_{0}\right|\right)>0$ and when $\left\|\beta_{d}\left(x_{d}\right)\right\| \neq 0,(18)$ gives $\frac{\partial P}{\partial \zeta}=2 \sqrt{\alpha_{d}\left(x_{d}\right)^{2}+\Omega\left(x_{d}\right) \beta_{d}\left(x_{d}\right) \Delta\left(x_{d}\right) \beta_{d}\left(x_{d}\right)^{T}}>0$ replacing $\zeta$ in (31) by the expression of $\gamma$ (since $\zeta=\gamma(x)$ is a solution of (30)). Therefore $\frac{\partial P}{\partial \zeta}$ never vanishes at each point of the form $\left\{\left(x_{d}, \gamma\left(x_{d}\right)\right) \mid x_{d} \in \mathcal{X}^{*}\right\}$. Furthermore, $P$ is smooth w.r.t. $x_{d}$ and $\zeta$ since so are $\alpha_{d}, \beta_{d}, \Omega$ and $\Delta$. Hence, using the implicit function theorem, $\gamma$ is smooth on $\mathcal{X}^{*}$.

The decrease of the CLKF of the form (5) when applying the event-based feedback (16)-(17) is easy to prove. For this, let consider the time interval $\left[t_{i}, t_{i+1}\right]$, that is the interval separating two successive events. Recall that $x_{d i}$ denotes the value of the state when the $i^{\text {th }}$ event occurs and $t_{i}$ the corresponding time instant, as defined in (15). At time $t_{i}$, when the event occurs, the time derivative of the CLKF, i.e. (7), after the update of the control is

$$
\frac{d V}{d t}\left(x_{d i}\right)=\alpha_{d}\left(x_{d i}\right)+\beta_{d}\left(x_{d i}\right) v\left(x_{d i}\right)=-\psi\left(x_{d i}\right)<0
$$

when substituting (18) in (16), where $\psi$ is defined in (29). More precisely, defining a compact set not containing the origin, that is $\Sigma=\left\{x_{d} \in C P([-r, 0], \mathcal{X})\right.$ : $\left.d \leq\left\|x_{d}\right\| \leq D\right\}$, where $C P([-r, 0], \mathcal{X})$ denotes the space of piecewise continuous functions from $[-r, 0]$ into $\mathcal{X}, d$ and $D$ are some constant in $\mathbb{R}^{+}$. If $V$ is a CLKF for the system of the form (4) then for all $0<\delta<D$ there exists $\varepsilon>0$ such that $\alpha_{d}\left(\chi_{d}\right) \geq-\frac{1}{2} \lambda\left(\left|\chi_{0}\right|\right) \Rightarrow\left|\beta_{d}\left(\chi_{d}\right)\right| \geq \varepsilon$ for $\chi_{d} \in \Sigma$. This gives

$$
\dot{V} \leq-\lambda(|x|)
$$

One can refer to Lemma 1 in [17], and [16], for further details. With this updated control, the event function (17) hence becomes strictly positive 


$$
\epsilon\left(x_{d i}, x_{d i}\right)=(1-\sigma) \psi\left(x_{d i}\right)>0
$$

since $\sigma \in[0,1[$, where $\psi$ is defined in (29). Furthermore, the event-function necessarily remains positive before the next event by continuity, because an event will occur when $\epsilon\left(x_{d}, x_{d i}\right)=0$ (see Definition 3). Therefore, on the interval $\left[t_{i}, t_{i+1}\right]$, one has

$$
\begin{aligned}
\epsilon\left(x_{d}, x_{d i}\right) & =-\alpha_{d}\left(x_{d}\right)-\beta_{d}\left(x_{d}\right) v\left(x_{d i}\right)-\sigma \psi\left(x_{d}\right) \\
& =-\frac{d V}{d t}\left(x_{d}\right)-\sigma \psi\left(x_{d}\right) \geq 0
\end{aligned}
$$

which ensures the decrease of the CLKF on the interval since $\sigma \psi\left(x_{d}\right) \geq 0$, where $\psi$ is defined in (29). Moreover, $t_{i+1}$ is necessarily bounded since, if not, $V$ should converge to a constant value where $\frac{d V}{d t}=0$, which is impossible thanks to the inequality above. The event function precisely prevents this phenomena detecting when $\frac{d V}{d t}$ is close to vanish and updates the control if it happens, where $\sigma$ is a tunable parameter fixing how "close to vanish" has to be the time derivative of $V$.

To prove that the event-based control is MSI, one has to prove that for any initial condition in an a priori given set, the sampling intervals are below bounded. First of all, notice that events only occur when $\epsilon$ becomes negative (with $x_{d} \neq 0$ ). Therefore, using the fact that when $\beta_{d}\left(x_{d}\right)=0, \alpha_{d}\left(x_{d}\right)<-\lambda\left(\left|\chi_{0}\right|\right)$ (because $V$ is a CLKF as defined in Definition 2), it follows from (17), on $\left\{x_{d} \in \mathcal{X}^{*} \mid\left\|\beta_{d}\left(x_{d}\right)\right\|=0\right\}$, that

$$
\epsilon\left(x_{d}, x_{d i}\right)=-\alpha_{d}\left(x_{d}\right)-\sigma\left|\alpha_{d}\left(x_{d}\right)\right|=(1-\sigma) \lambda\left(\left|\chi_{0}\right|\right)>0
$$

because $\sigma \in[0,1[$ and $\lambda(s)>0$ for $s>0$. Therefore, there is no event on the set $\left\{x_{d} \in \mathcal{X} \mid\left\|\beta_{d}\left(x_{d}\right)\right\|=0\right\} \cup\{0\}$. The study is then restricted to the set $\mathcal{S}_{d}^{*}=\left\{x_{d} \in\right.$ $\left.\mathcal{X}^{*} \mid\left\|\beta_{d}\left(x_{d}\right)\right\| \neq 0\right\}$, where $\Omega$ and $\Delta$ are strictly positive by assumption. Rewriting the time derivative of the CLKF along the trajectories yields

$$
\begin{aligned}
\frac{d V}{d t}\left(x_{d}\right) & =\alpha_{d}\left(x_{d}\right)+\beta_{d}\left(x_{d}\right) v\left(x_{d i}\right) \\
& =-\psi\left(x_{d}\right)+\beta_{d}\left(x_{d}\right)\left(v\left(x_{d i}\right)-v\left(x_{d}\right)\right)
\end{aligned}
$$

when using the definition of $v\left(x_{d}\right)$ in (16) and (18), where $\psi$ is defined in (29). Let respectively define the level and the set

$$
\begin{aligned}
& \vartheta_{i}:=V\left(x_{d i}\right) \quad \forall x_{d i} \in \mathcal{S}_{d} \\
& \mathcal{V}_{\vartheta_{i}}:=\left\{x_{d} \in \mathcal{X} \mid V\left(x_{d}\right) \leq \vartheta_{i}\right\}
\end{aligned}
$$

From the choice of the event function, it follows from (32) that $x_{d}$ belongs to $\mathcal{V}_{\vartheta} \subset \mathcal{V}_{\vartheta_{i}}$. Note that if $x_{d i}$ belongs to $\mathcal{S}_{d}$, this is not necessarily the case for $x_{d}$ that can escape from this set. First see that, since i) $\Omega\left(x_{d}\right)$ is such that $\alpha_{d}\left(x_{d}\right)^{2}+$ $\Omega\left(x_{d}\right) \beta_{d}\left(x_{d}\right) \Delta\left(x_{d}\right) \beta_{d}\left(x_{d}\right)^{T}>0$ for all $x_{d} \in \mathcal{S}_{d}^{*}$, and ii) $\alpha_{d}\left(x_{d}\right)$ is necessarily non-zero on the frontier of $\mathcal{S}_{d}$ (except possibly at the origin) 


$$
\frac{d V}{d t}\left(x_{d i}\right)=-\psi\left(x_{d i}\right) \leq-\inf _{\substack{x_{d i} \in \mathcal{S}_{d} \\ \text { s.t. } V\left(x_{d i}\right)=\vartheta_{i}}} \psi\left(x_{d i}\right)=:-\varphi\left(\vartheta_{i}\right)<0
$$

Considering now the second time derivative of the CLKF

$$
\begin{aligned}
& \ddot{V}\left(x_{d}\right)=\left(\frac{\partial \alpha_{d}}{\partial x_{d}}\left(x_{d}\right)+v\left(x_{d i}\right)^{T} \frac{\partial \beta_{d}^{T}}{\partial x_{d}}\left(x_{d}\right)\right) \Theta\left(x_{d}, x_{d i}\right) \\
& \text { with } \Theta\left(x_{d}, x_{d i}\right):=\Phi\left(x_{\tau}\right)+g\left(x_{\tau}\right) v\left(x_{d i}\right)
\end{aligned}
$$

where $\Phi$ is defined in (4). By continuity of all the involved functions (except for $\Gamma$ in $\Phi$ which is piecewise continuous but bounded by assumption), both terms can be bounded for all $x_{d} \in \mathcal{V}_{\vartheta_{i}}$ by the following upper bounds $\varrho_{1}\left(\vartheta_{i}\right)$ and $\varrho_{2}\left(\vartheta_{i}\right)$ such that

$$
\begin{aligned}
& \varrho_{1}\left(\vartheta_{i}\right):=\sup _{x_{d i} \in \mathcal{S}_{d}{\operatorname{s.t.~} V\left(\mathcal{V}_{d i}\right)=\vartheta_{i}}_{x_{d} \in \mathcal{V}_{\vartheta_{i}}}}\left\|\frac{\partial \alpha_{d}}{\partial x_{d}}\left(x_{d}\right)+v\left(x_{d i}\right)^{T} \frac{\partial \beta_{d}^{T}}{\partial x_{d}}\left(x_{d}\right)\right\| \\
& \varrho_{2}\left(\vartheta_{i}\right):=\sup _{x_{d i} \in \mathcal{S}_{d}{\operatorname{s.t.~} V\left(x_{d i}\right)=\vartheta_{i}}_{x_{d} \in \mathcal{V}_{\vartheta_{i}}}}\left\|\Theta\left(x_{d}, x_{d i}\right)\right\|
\end{aligned}
$$

where $\Theta$ is defined in (34). Therefore, $\dot{V}$ is strictly negative at any event instant $t_{i}$ and cannot vanish until a certain time $\underline{\tau}\left(\vartheta_{i}\right)$ is elapsed (because its slope is positive). This minimal sampling interval is only depending on the level $\vartheta_{i}$. A bound on $\underline{\tau}\left(\vartheta_{i}\right)$ is given by the inequality

$$
\frac{d V}{d t}\left(x_{d}\right) \leq \frac{d V}{d t}\left(x_{d i}\right)+\rho_{1} \rho_{2}\left(t-t_{i}\right) \quad \forall x_{d} \in \mathcal{V}_{\vartheta_{i}}
$$

that yields

$$
\underline{\tau}\left(\vartheta_{i}\right) \geq \frac{\varphi\left(\vartheta_{i}\right)}{\varrho_{1}\left(\vartheta_{i}\right) \varrho_{2}\left(\vartheta_{i}\right)}>0
$$

where $\varphi$ is defined in (33). As a consequence, the event-based feedback (16)-(17) is semi-uniformly MSI. This ends the proof of Theorem 3.

\section{Proof of Property 4}

To prove the continuity of $v$ at the origin, one only needs to consider the points in $\mathcal{S}$ since $v\left(x_{d}\right)=0$ if $\left\|\beta_{d}\left(x_{d}\right)\right\|=0$. Then (16) gives 


$$
\begin{aligned}
\left\|v\left(x_{d}\right)\right\| \leq & \frac{\left|\alpha_{d}\left(x_{d}\right)\right|}{\beta_{d}\left(x_{d}\right) \Delta\left(x_{d}\right) \beta_{d}\left(x_{d}\right)^{T}}\left\|\Delta\left(x_{d}\right) \beta_{d}\left(x_{d}\right)^{T}\right\| \\
& +\frac{\psi\left(x_{d}\right)}{\beta_{d}\left(x_{d}\right) \Delta\left(x_{d}\right) \beta_{d}\left(x_{d}\right)^{T}}\left\|\Delta\left(x_{d}\right) \beta_{d}\left(x_{d}\right)^{T}\right\| \\
\leq & \frac{2\left|\alpha_{d}\left(x_{d}\right)\right|}{\beta_{d}\left(x_{d}\right) \Delta\left(x_{d}\right) \beta_{d}\left(x_{d}\right)^{T}}\left\|\Delta\left(x_{d}\right) \beta_{d}\left(x_{d}\right)^{T}\right\| \\
& +\sqrt{\Omega\left(x_{d}\right)\left\|\Delta\left(x_{d}\right)\right\|}
\end{aligned}
$$

With the small control property (see Property 1), for any $\varepsilon>0$, there is $\mu>0$ such that for any $x_{d} \in \mathcal{B}(\mu) \backslash\{0\}$, there exists some $u$ with $\|u\| \leq \varepsilon$ such that $L_{f}^{*} V\left(x_{d}\right)+\left[L_{g} V_{1}\left(x_{d}\right)\right]^{T} u=\alpha_{d}\left(x_{d}\right)+\beta_{d}\left(x_{d}\right) u<0$ and therefore $\left|\alpha_{d}\left(x_{d}\right)\right|<$ $\left\|\beta_{d}\left(x_{d}\right)\right\| \varepsilon$. It follows

$$
\left\|v\left(x_{d}\right)\right\| \leq \frac{2 \varepsilon\left\|\beta_{d}\left(x_{d}\right)\right\|\left\|\Delta\left(x_{d}\right) \beta_{d}\left(x_{d}\right)^{T}\right\|}{\beta_{d}\left(x_{d}\right) \Delta\left(x_{d}\right) \beta_{d}\left(x_{d}\right)^{T}}+\sqrt{\Omega\left(x_{d}\right)\left\|\Delta\left(x_{d}\right)\right\|}
$$

Since the function $\left(v_{1}, v_{2}\right) \rightarrow \frac{\left\|v_{1}\right\|\left\|v_{2}\right\|}{v_{1}^{T} v_{2}}$ is continuous w.r.t. its two variables at the origin where it equals 1 , since $\Omega$ and $\Delta$ are also continuous, since $\Omega\left(x_{d}\right)\left\|\Delta\left(x_{d}\right)\right\|$ vanishes at the origin, for any $\varepsilon^{\prime}$, there is some $\mu^{\prime}$ such that $\forall x_{d} \in \mathcal{B}\left(\mu^{\prime}\right) \backslash\{0\}$, $\left\|v\left(x_{d}\right)\right\| \leq \varepsilon^{\prime}$ which ends the proof of continuity.

\section{Proof of Property 5}

With $\Omega$ defined as in (20), the feedback in (16) becomes

$$
v\left(x_{d}\right)=-\beta_{d}\left(x_{d}\right) \Delta\left(x_{d}\right) \omega\left(x_{d}\right)
$$

if the condition (19) is satisfied, which is obviously smooth on $\mathcal{X}$. Note that the expression of $\Omega$ in (20) comes from the solution of (30), where $\omega$ only has to be smooth.

\section{References}

1. A. Anta and P. Tabuada. To sample or not to sample: Self-triggered control for nonlinear systems. Voluntary Action Leadership, 55:2030-2042, 2010.

2. Z. Artstein. Stabilization with relaxed controls. In Nonlinear Analysis: Theory, Methods \& Applications, 7:1163-1173, 1983.

3. K. E. Årzén. A simple event-based PID controller. In Preprints of the 14th World Congress of IFAC, 1999.

4. K. J. Åström and B. Bernhardsson. Comparison of Riemann and Lebesque sampling for first order stochastic systems. In Proceedings of the 41st IEEE Conference on Decision and Control, 2002. 
5. J. Curtis and R. Beard. Satisficing: A new approach to constructive nonlinear control. IEEE Transactions on Automatic Control, 49:1090-1102, 2004.

6. S. Durand. Event-based stabilization of linear system with communication delays in the measurements. In Proceedings of the American Control Conference, 2013.

7. S. Durand and N. Marchand. Further results on event-based PID controller. In Proceedings of the European Control Conference, 2009.

8. S. Durand, N. Marchand, and J. F. Guerrero Castellanos. Event-based stabilization of nonlinear time-delay systems. In Proceedings of the 19th World Congress of IFAC, 2014.

9. A. Eqtami, D. V. Dimarogonas, and K. J. Kyriakopoulos. Event-triggered control for discretetime systems. In Proceedings of the IEEE American Control Conference, 2010.

10. R. Freeman and P. Kokotovic. Robust Nonlinear Control Design: State-Space and Lyapunov Techniques. Birkhäuser Basel, 1996.

11. E. Fridman. Introduction to time-delay and sampled-data systems. In Proceedings of the European Control Conference, 2014.

12. K. Gu and S. Niculescu. Survey on recent results in the stability and control of time-delay systems. Journal of Dynamic Systems, Measurement, and Control, 125:158-165, 2003.

13. M. Guinaldo, D. Lehmann, J. Sánchez, S. Dormido, and K. H. Johansson. Distributed eventtriggered control with network delays and packet losses. In Proceedings of the 51st IEEE Conference on Decision and Control, 2012.

14. W. P. M. H. Heemels, J. H. Sandee, and P. P. J. van den Bosch. Analysis of event-driven controllers for linear systems. International journal of control, 81:571-590, 2009.

15. P. Hsu and S. Sastry. The effect of discretized feedback in a closed loop system. In Proceedings of the 26th IEEE Conference on Decision and Control, 1987.

16. M. Jankovic. Control Lyapunov-Razumikhin functions to time-delay systems. In Proceedings of the 38th IEEE Conference on Decision and Control, 1999.

17. M. Jankovic. Extension of control Lyapunov functions to time-delay systems. In Proceedings of the 39th IEEE Conference on Decision and Control, 2000.

18. M. Jankovic. Control of nonlinear systems with time-delay. In Proceedings of the 42th IEEE Conference on Decision and Control, 2003.

19. H. Khalil. Nonlinear Systems, Third Edition. Prentice Hall, 2002.

20. N. Krasowskii. Stability of motion (in Russian). Stanford University Press, 1963.

21. D. Lehmann and J. Lunze. Event-based control with communication delays. In Proceedings of the 18th IFAC world congress, 2011.

22. D. Lehmann and J. Lunze. Event-based control with communication delays and packet losses. International Journal of Control, 85:563-577, 2012.

23. J. Lunze and D. Lehmann. A state-feedback approach to event-based control. Automatica, 46:211-215, 2010.

24. N. Marchand, S. Durand, and J. F. Guerrero-Castellanos. A general formula for event-based stabilization of nonlinear systems. IEEE Transactions on Automatic Control, 58(5):13321337, 2013.

25. D. Nešić and L. Grüne. Lyapunov-based continuous-time nonlinear controller redesign for sampled-data implementation. Automatica, 41(7):1143-1156, 2005.

26. D. Nešić and A. Teel. A framework for stabilization of nonlinear sampled-data systems based on their approximate discrete-time models. IEEE Transactions on Automatic Control, 49(7):1103-1122, 2004.

27. A. Papachristodoulou, M. Peet, and S. Lall. Constructing Lyapunov-Krasovskii functionals for linear time delay systems. In Proceedings of the 2005 American Control Conference, 2005.

28. B. Razumikhin. On the stability of systems with a delay (in russian). Journal of Applied Mathematics and Mechanics (Translation of Prikl. Mat. Mekh.), 1956.

29. J. Sánchez, M. Guarnes, and S. Dormido. On the application of different event-based sampling strategies to the control of a simple industrial process. Sensors, 9:6795-6818, 2009.

30. J. H. Sandee, W. P. M. H. Heemels, and P. P. J. van den Bosch. Event-driven control as an opportunity in the multidisciplinary development of embedded controllers. In Proceedings of the American Control Conference, pages 1776-1781, 2005. 
31. R. Sepulchre, M. Jankovic, and P. Kokotovic. Constructive Nonlinear Control. SpringerVerlag, 1997.

32. E. D. Sontag. A Lyapunov-like characterization of asymptotic controllability. In SIAM Journal on Control and Optimization, 21:462-471, 1983.

33. E. D. Sontag. A "universal" construction of Artstein's theorem on nonlinear stabilization. Systems \& Control Letters, 13:117-123, 1989.

34. P. Tabuada. Event-triggered real-time scheduling of stabilizing control tasks. IEEE Transactions on Automatic Control, 52:1680-1685, 2007.

35. M. Velasco, P. Martí, and E. Bini. On Lyapunov sampling for event-driven controllers. In Proceedings of the 48th IEEE Conference on Decision and Control, 2009. 